\title{
The Relationships Between Uncertainty and Coping Behaviors of Patients With Liver Cirrhosis
}

\author{
Wan-Ju Lin ${ }^{1}$, Hui-Man Huang ${ }^{2 *}$, Bih-O Lee ${ }^{3}$ and Fan-Ko Sun ${ }^{4}$ \\ ${ }^{1}$ Emergency Department, Sin Lau Hospital No. 57, Sec. 1, Dongmen Rd., East Dist., Tainan City 70142, Taiwan \\ ${ }^{2}$ Department of Nursing, National Quemoy University, No. 1, University Rd. Jinning Township, Kinmen County 89250, Taiwan \\ ${ }^{3}$ Department of Nursing, Chang Gung University of Science and Technology, Chiayi Campus, Puzi City, Chiayi County 61363, Taiwan \\ ${ }^{4}$ Department of Nursing, I-Shou University, No.8, Yida Rd., Jiaosu Village, Yanchao District, Kaohsiung City 82445, Taiwan
}

\section{Abstract}

Background: Patients tend to develop a sense of uncertainty when they lack control over the progress of the disease, and physical discomforts. The sense of uncertainty that patients develop when facing their disease or treatment may elicit physical and psychological distress which can influence the patients' coping behaviors.

Purpose: This study investigated the relationships between cirrhotic patients' sense of uncertainty and coping behaviors.

Methods: A cross-sectional, correlational research design was used. Convenience sampling was performed to select 76 cirrhotic inpatients in the division of gastroenterology at a hospital in Southern Taiwan. Three structured questionnaires (Personal Characteristics Questionnaire, Mishel Uncertainty in Illness Scale, Coping Behaviors Scale) were used for data collection.

Results: (a) Cirrhotic patients perceived a moderate or increased level of uncertainty and problemoriented coping behaviors were the most frequently used coping behaviors. (b) Patients who had no religion, who had fatigue or severe disease conditions exhibited comparatively high levels of uncertainty. (c) Patients who were singled or unemployed, or fatigue often showed emotion-oriented coping behaviors. Consuming alcohol frequency, illness severity was positively correlated with emotionoriented coping behaviors. (d) Patients who were unemployed often exhibited problem-oriented coping behaviors. Educational level and consuming alcohol frequency were positively correlated with problemoriented coping behaviors. (e) Patients' sense of uncertainty was positively correlated with emotion- and problem-oriented coping behaviors.

Conclusions/ Implications for Practice: Since cirrhotic patients frequently exhibit emotion-oriented coping behaviors and have high level of uncertainty, nurses could encourage them to express their emotions and problems, and listen to them patiently. If patients were high levels of uncertainty, and exhibit problem-oriented coping behaviors, by spontaneously providing disease-related information and social support, they can readily adopt to their disease conditions.

\section{Introduction}

Chronic liver disease and cirrhosis rank as the ninth among the ten leading causes of death in Taiwan. The number of cirrhosis-induced deaths has increased annually from 3,075 in 1981 to 4,922 in 2012 [1]. Patients with cirrhosis experience physiological distress caused by the disease, which in turn leads to mental health problems [2]. During the progression of a disease, patients frequently sense a lack of control over the participation in treatment decision making, the improvement of physical discomfort, the entire treatment process, and the acquisition of medical information. When people perceive a lack of control over matters that concern them, they tend to develop a sense of uncertainly that impacts and disturbs themselves mentally and physically [3]. Coping behavior refers to a dynamic process of selfadjustment, in which individuals alter their cognition and behavior in response to a stressful situation to alleviate their mental and physical burden [4]. Ko and Shu [5] reported that a strong sense of uncertainty reduces gynecologic inpatients' ability to clarify questions and seek resources, thereby affecting their ability to cope with and adapt to the disease and treatment.

\section{Literature Review}

According to studies that have explored the factors affecting uncertainty, patients' personal characteristics including age, education

\section{Publication History:}

Received: September 08, 2017 Accepted: November 22, 2017 Published: November 24, 2017

\section{Keywords:}

Cirrhotic patients, Sense of uncertainty, Emotion-oriented coping behaviors, Problem-oriented coping behaviors level, and religion are related to feelings of uncertainty. Older patients perceive lower level of uncertainty [6], whereas patients with a low level of education experience a stronger sense of uncertainty [7]. Nonreligious patients demonstrate a significantly higher level of uncertainty than do religious patients [8]. Moreover, studies investigating the factors affecting coping behaviors have confirmed that age, education level, marital status, religion, and employment status are related to coping behavior. Specifically, age correlates positively with the frequency of engaging in problem-oriented coping behavior [9]. Patients with a higher level of education tend to adopt problem-oriented coping behavior rather than emotionoriented coping behavior, and they frequently seek social resources to solve problems $[9,10]$. Unmarried or widowed patients with chronic disease mostly live alone and tend to engage in emotion-oriented coping behavior because of a weaker family support system [11].

"Corresponding Author: Dr. Hui-Man Huang, Department of Nursing, National Quemoy University, No. 1, University Rd. Jinning Township, Kinmen County 89250, Taiwan, ROC, Tel: + 886-956070508; E-mail: x2156@nqu.edu.tw

Citation: Lin WJ, Huang HM, Lee BO, Sun FK (2017) The Relationships Between Uncertainty and Coping Behaviors of Patients With Liver Cirrhosis. Int J Nurs Clin Pract 4: 261. doi: https://doi.org/10.15344/2394-4978/2017/261

Copyright: ( 2017 Lin et al. This is an open-access article distributed under the terms of the Creative Commons Attribution License, which permits unrestricted use, distribution, and reproduction in any medium, provided the original author and source are credited. 
Religious patients more frequently adopt problem-oriented coping behavior than do nonreligious patients [9]. However, this result was inconsistent with the findings presented by Tsui, Lin, \& Huang [12] Liou et al. [9] observed that unemployed patients more often engage in emotion-oriented coping behavior, whereas patients who have a job typically apply problem-oriented coping strategies.

In summary, previous studies have presented inconclusive results regarding the factors affecting patients' level of uncertainty and adoption of coping behaviors. Most of these studies have focused on patients with cancer [6], heart disease [10], and dialysis-dependent disease [13], as well as on their primary caregivers [14]. In addition, past studies on patients with cirrhosis generally focus on the quality of life of these patients [15], seldom exploring their feelings of uncertainty and coping behaviors. In order to assist nurses in understanding the perceptions of patients with cirrhosis so that they could provide individualized nursing interventions to these patients. Therefore, the purposes of this study were to investigate the personal characteristics of patients with cirrhosis, level of uncertainty, coping behaviors, and the relationships among them.

\section{Method}

This study adopted a cross-sectional research design and face-toface interview to collect data of patients with cirrhosis.

\section{Sample}

Convenience sampling was used with seventy-eight eligible volunteers in a regional hospital agreed to participate. Two of the volunteers withdrew during the interview because they felt too fatigue. Therefore, the total sample included 76 participants. Patients with a history of cancer, cardiac cirrhosis, primary biliary cirrhosis, or hepatic encephalopathy with a severe coma were excluded. The inclusion criteria of this study were (a) age of 20-64 years and a diagnosis of cirrhosis; (b) no severe mental disorders; and (c) ability to communicate in words, Mandarin Chinese or Taiwanese Hokkien. The authors of this study employed G-power 3.1 to calculate the number of samples required for correlation analysis, with test family set as exact, statistical test as correlation, and $\alpha=.05$. The estimated number of samples was 67 when power was set as .8, correlation $\mathrm{H} 1$ as a moderate effect size of 0.3 , and correlation $\mathrm{H} 0$ as 0 . The actual number of valid samples in this study was 76 (power $>.85$ ), indicating a satisfactory statistical power of test.

\section{Instruments}

\section{Personal characteristics questionnaire}

The content of the questionnaire included: age, education level, religion, marital status, employment status, frequency of alcohol intake, disease severity, and level of fatigue. The Child-Pugh score (515) was used to measure disease severity, and the scores were graded into three levels: Child A (5-6; mild), Child B (7-9; moderate), and Child C $(\geq 10$; severe).

\section{Mishel uncertainty in illness scale (Chinese version)}

The Mishel Uncertainty in Illness Scale (Chinese version), translated and revised by Sheu and Hwang [7], was adopted, and the content of the scale involved uncertainty factors and complexity factors. Lai et al. [6] employed the original 25-item scale to explore the level of uncertainty among patients with liver cancer and invited two experts to review the scale. The experts removed two items from the scale because patients with liver cancer were confirmed of their diagnosis, informed of their conditions, and under treatment. This 23-item uncertainty in illness scale was used in the present study, and the scale had a Cronbach's $\alpha$ of .78. Items 6, 7, 9, 22, and 23 of this scale were negatively worded and scored. A 5-point Likert scale was adopted with scores ranging from 1 (strongly disagree) to 5 (strongly agree). Higher total scores indicated higher levels of uncertainty (range of total score $=23-115$ ). The Cronbach's $\alpha$ of the entire scale obtained during the formal research was .87 , indicating that this scale exhibited satisfactory internal consistency.

\section{Coping behaviors scale(Chinese version)}

The Coping Behaviors Scale (Chinese version) translate and revised by Chiou [13] was adopted in this study (content validity index $=.85$; Cronbach's $\alpha=.78$ ). This scale contained a total of 40 items and was divided into two dimensions, with 15 items measuring problem-oriented coping behavior and 25 items measuring emotionoriented coping behavior. A 5-point Likert scale was employed, with scores ranging from 1 (never) to 5 (always). Higher scores indicated a higher frequency of using a particular type of coping behavior. The Cronbach's $\alpha$ of the overall scale obtained during the formal research was .76, and the Cronbach's a values for the dimensions of problemoriented and emotion-oriented coping behaviors were .81 and .74 , respectively, indicating satisfactory internal consistency.

\section{Ethical considerations}

This study was approved by the institutional review board of the hospital (IRB No. 14-015) where the research participants were recruited. Before signing a consent form, participants were informed of the research objectives, procedures, and testing methods of this study and were guaranteed the freedom to withdraw from the research at any time.

\section{Data analysis}

The SPSS Windows 16.0 was employed for data analysis, and statistical analyses including percentage, mean, standard deviation, $t$-test, one-way ANOVA, and Pearson product-moment correlation were performed.

\section{Results}

\section{Personal characteristics, uncertainty and coping behaviors}

In this study, the patients with cirrhosis were 34-63 years old (mean age of $48.72 \pm 8.16$ years). Male participants $(84.2 \%)$ outnumbered female participants (15.8\%). A majority of the participants had a moderate level of education or above (average years of education = $11.12 \pm 2.90)$ and were single $(n=43 ; 56.6 \%)$. Taoist believers accounted for the largest percentage $(n=34 ; 44.7 \%)$ of the total participants. Moreover, most of the participants were unemployed $(n=42 ; 55.3 \%)$ and consumed alcohol regularly $(n=60 ; 78.9 \%)$. Regarding disease severity, most participants were categorized as Child A $(n=35 ; 46.0 \%)$ according to the Child-Pugh score, and 68 participants (89.5\%) experienced fatigue (Table 1). As shown in Table 2, the participants of this study perceived a moderate level of uncertainty (mean score of $71.81 \pm 10.6)$. Emotion-oriented and problem-oriented coping behaviors obtained a mean score of $67.38 \pm 11.03(\beta=53.90)$ and 45.69 $\pm 7.07(\beta=60.92)$, respectively. These values revealed that cirrhotic patients more often engage in problem-oriented coping behavior rather than emotion-oriented coping behavior. 


\begin{tabular}{|c|c|c|c|c|c|c|}
\hline Variable & $\mathrm{M} \pm \mathrm{SD}$ & n (\%) & & \multicolumn{2}{|c|}{ Variable } & n (\%) \\
\hline Age & $48.72 \pm 8.16$ & $76(100)$ & & \multicolumn{2}{|c|}{ Employment } & \\
\hline Gender & & & & \multicolumn{2}{|c|}{ No } & $42(55.3)$ \\
\hline Male & & $64(84.2)$ & & \multicolumn{2}{|c|}{ Yes } & $34(44.7)$ \\
\hline Female & & $12(15.8)$ & & \multicolumn{2}{|c|}{ Alcohol intake frequency } & \\
\hline Education level & $11.12 \pm 2.90$ & & & \multicolumn{2}{|c|}{ Do not drink } & $16(21.1)$ \\
\hline$<$ Junior high school & & $32(42.1)$ & & \multicolumn{2}{|c|}{ Drink } & $60(78.9)$ \\
\hline$\geq$ Junior high school & & $44(57.9)$ & & \multicolumn{2}{|c|}{ Quit drinking } & $12(15.8)$ \\
\hline Marital status & & & & \multicolumn{2}{|c|}{ Seldom } & $24(31.6)$ \\
\hline Single & & $43(56.6)$ & & \multicolumn{2}{|c|}{ Frequently } & $16(21.1)$ \\
\hline Unmarried & & $27(35.5)$ & & \multicolumn{2}{|c|}{ Every day } & $8(10.5)$ \\
\hline Divorced & & $13(17.1)$ & & \multicolumn{2}{|c|}{ Disease severity } & \\
\hline Widowed & & $3(4.0)$ & & \multicolumn{2}{|c|}{ Child A } & $35(46.0)$ \\
\hline Married & & $33(43.4)$ & & \multicolumn{2}{|c|}{ Child B } & $29(38.2)$ \\
\hline Religion & & & & \multicolumn{2}{|c|}{ Child C } & $12(15.8)$ \\
\hline None & & $21(27.6)$ & & \multicolumn{2}{|c|}{ Fatigue } & \\
\hline Yes & & $55(72.4)$ & & \multicolumn{2}{|c|}{ No } & $8(10.5)$ \\
\hline Buddhism & & $11(14.5)$ & & \multirow{3}{*}{\multicolumn{2}{|c|}{ Yes }} & $68(89.5)$ \\
\hline Christianity & & $10(13.2)$ & & & & \\
\hline Taoism & & $34(44.7)$ & & & & \\
\hline \multicolumn{7}{|c|}{$\begin{array}{l}\text { Table 1: Personal characteristics of patients with cirrhosis }(\mathrm{N}=76) \text {. } \\
\text { Note: Range of age }=34-63 \text { years-old }\end{array}$} \\
\hline Item & n (\%) & $\mathrm{M} \pm \mathrm{SD}$ & Score & $\beta$ score $(\%)$ & Minimum & Maximum \\
\hline Overall level of uncertainty & & $71.81 \pm 10.67$ & & 62.44 & 52 & 101 \\
\hline Uncertainty & & $44.03 \pm 7.49$ & $13-65$ & 67.73 & 29 & 64 \\
\hline Complexity & & $27.79 \pm 4.15$ & $10-50$ & 55.58 & 19 & 38 \\
\hline Mild & $3(4.0)$ & & $23-53$ & & & \\
\hline Moderate & $64(84.2)$ & & $54-83$ & & & \\
\hline Severe & $9(11.8)$ & & $84-113$ & & & \\
\hline \multicolumn{7}{|l|}{ Coping behaviors } \\
\hline Emotion-oriented & & $67.38 \pm 11.03$ & 44- 93 & 53.90 & 93 & 44 \\
\hline Problem-oriented & & $45.69 \pm 7.07$ & $30-59$ & 60.92 & 59 & 30 \\
\hline
\end{tabular}

Table 2: Uncertainty and coping behaviors of patients with cirrhosis $(\mathrm{N}=76)$.

Note: $\beta$ score $(\%)=$ mean score / total score $\times 100 \%$

\section{Relationships between the variables of personal characteristics and uncertainty}

Table 3 reveals that the overall level of uncertainty in patients with cirrhosis differed significantly according to whether the patients believed in a religion or experienced fatigue. Furthermore, disease severity correlated significantly and positively with the overall level of uncertainty, and cirrhotic patients who were classified into Child B and Child C sensed a significantly higher level of uncertainty than did those who were categorized as Child A.

Relationships among the variables of personal characteristics, uncertainty and coping behaviors

According to Table 4, the frequency patients with cirrhosis adopt emotion-oriented coping behavior differed significantly with their marital status, employment status, and perception of fatigue. The patients' frequency of alcohol intake and disease severity correlated significantly and positively with emotion-oriented coping behavior. The frequency of using problem-oriented coping behavior differed significantly according to the patients' employment status. The level of education and frequency of alcohol intake correlated significantly and positively with problem-oriented coping behavior. Furthermore, the overall level of uncertainty in patients with cirrhosis correlated significantly and positively with emotion-oriented and problemoriented coping behaviors (Table 4).

\section{Discussion}

\section{Personal characteristics and the uncertainty}

The results of this study showed that the overall level of uncertainty was higher in nonreligious patients with cirrhosis compared with those who had a religion. This finding corresponded with the results presented by Yang et al. [16]. Believing in a religion serves to ease a person's mind, answer doubts in life, and give hope and affirmation. 


\begin{tabular}{|c|c|c|c|c|c|}
\hline \multirow[t]{2}{*}{ Variable } & \multirow[t]{2}{*}{$\mathrm{n}$} & \multicolumn{4}{|c|}{ Overall level of uncertainty } \\
\hline & & $\mathrm{M} \pm \mathrm{SD}$ & $t / F$ & $\mathbf{P}$ & $\mathbf{r}$ \\
\hline Religion & & & -2.00 & $.048^{\star}$ & \\
\hline None & 21 & $75.71 \pm 9.14$ & & & \\
\hline Yes & 55 & $70.71 \pm 10.91$ & & & \\
\hline Disease severity & & & 13.8 & $.000^{* * *}$ & $0.532^{\star * *}$ \\
\hline a. & Child A & 35 & $66.29 \pm 8.42$ & & \\
\hline b. & Child B & 29 & $74.62 \pm 10.36$ & & \\
\hline c. & Child C & 12 & $81.70 \pm 8.43$ & & \\
\hline Post-hoc comparison & & & $\mathrm{b}>\mathrm{a} ; \mathrm{c}>\mathrm{a}$ & & \\
\hline Fatigue & & & 2.29 & $.025^{*}$ & \\
\hline No & 8 & $63.88 \pm 8.06$ & & & \\
\hline Yes & 68 & $72.75 \pm 10.60$ & & & \\
\hline
\end{tabular}

\begin{tabular}{|c|c|c|c|c|c|c|c|}
\hline & & $\begin{array}{l}\text { Emotion-oriented } \\
\text { coping behavior }\end{array}$ & & & $\begin{array}{l}\text { Problem-oriented } \\
\text { coping behavior }\end{array}$ & & \\
\hline Variable & $\mathbf{n}$ & $\mathrm{M} \pm \mathrm{SD}$ & $t / F$ & $\mathbf{p}$ & $\mathrm{M} \pm \mathrm{SD}$ & $t / F$ & $\mathbf{p}$ \\
\hline Marital status & & & 2.93 & $.005^{\star *}$ & & 0.521 & .594 \\
\hline Single & 43 & $70.30 \pm 12.49$ & & & $46.07 \pm 7.67$ & & \\
\hline Married & 33 & $63.58 \pm 7.36$ & & & $45.21 \pm 6.29$ & & \\
\hline Employment & & & -2.14 & $.036^{*}$ & & -2.13 & $.037^{*}$ \\
\hline No & 42 & $69.76 \pm 11.47$ & & & $47.21 \pm 6.30$ & & \\
\hline Yes & 34 & $64.44 \pm 9.85$ & & & $43.82 \pm 7.60$ & & \\
\hline Fatigue & & & -2.64 & $.010^{\star *}$ & & -1.58 & .119 \\
\hline No & 8 & $58.00 \pm 6.14$ & & & $42.00 \pm 6.05$ & & \\
\hline Yes & 68 & $68.48 \pm 10.98$ & & & $46.13 \pm 7.10$ & & \\
\hline Variable & & $\begin{array}{l}\text { Emotion-oriented } \\
\text { coping behavior }(r)\end{array}$ & & & $\begin{array}{l}\text { Problem-oriented } \\
\text { coping behavior }(\mathrm{r})\end{array}$ & & \\
\hline Education level & & -0.275 & & & $0.314^{\star *}$ & & \\
\hline Alcohol intake frequency & & $0.470^{\star * *}$ & & & $0.426^{\star * *}$ & & \\
\hline Disease severity & & $0.334^{\star *}$ & & & 0.213 & & \\
\hline Overall level of uncertainty & & $0.459^{\star * *}$ & & & $0.372^{\star *}$ & & \\
\hline
\end{tabular}

Table 4: Difference/correlation analyses of coping behaviors among cirrhotic patients with different personal characteristics $(\mathrm{N}=76)$.

Note: 1 . Only significant variables are presented in this table $\left({ }^{*} \mathrm{p}<.05 ;{ }^{* *} \mathrm{p}<.01 ;{ }^{* * *} \mathrm{p}<.001\right)$.

Devoting to a religion enables patients to achieve spiritual sustenance, obtain a support they could depend on in life, and reflect on their life experiences [17]. Lin, Liou, and Chen [18] indicated that people pursue spiritual support when faced with pressure and adversity; they seek internal peace and comfort through the support of religion. Accordingly, this study infers that patients without religious beliefs experienced a higher level of uncertainty than do their counterparts possibly because they lacked spiritual support and perceived no sense of dependency.

The overall level of uncertainty was higher in patients with fatigue than in those without fatigue. This study found that numerous participants discontinued working or even resigned because they had to be hospitalized repeatedly for symptom recurrence. Tsao [19] noted that because patients with cirrhosis are prone to fatigue and thus need abundant rest, those with a job have to suspend their work or are forced to leave their positions. Consequently, these patients often experience difficulties in adapting to different roles and problems such as anxiety and loss of control over their future. Moreover, increased level of fatigue restricts patients' ability to undertake daily activities. Oftentimes, patients are uncertain about when they will be able to take care of themselves, what they can do, whether the treatment will be effective, and how they can plan their future. Because of these thoughts, the participants experienced a high level of uncertainty.

Patients' disease severity correlated significantly and positively with the overall level of uncertainty. This result was consistent with that of Yang and Chang [8], who found that symptom recurrence and 
repeated hospitalization increase patients' level of uncertainty. For patients with cirrhosis, severe disease leads to more complications, resulting in repeated hospitalization. These patients cannot know of their prognosis, which further elevates the level of uncertainty in them.

\section{Correlation between personal characteristics and emotion- oriented coping behaviors}

Cirrhotic patients who are single adopt emotion-oriented coping behavior more frequently than do those who are married. This result differed from that of Tsui et al. [12]. Zheng, Hsiao, and Chang [20] indicated that patients can acquire resources, encouragement, and vent their emotions through external support systems (e.g., partner, family, and friends) to cope with undesired incidents and reduce stress. Cirrhotic patients who are single lack a support system; therefore, their coping abilities are compromised due to symptom distress and difficulties in subsequent treatment and care processes. Chiou [13] also noted that people without a satisfactory support system are incapable of coping with stress. Lai [6] reported that patients with cancer mostly receive psychological support from their spouse. Therefore, the present study infers that cirrhotic patients who are single cannot effectively cope with the disease, because they lack psychological support from their spouse. Consequently, these patients have a poorer coping ability than do married patients, which thereby influences their attitude and compliance toward receiving treatment. Miller [4] also mentioned that a satisfactory support system can help patients adapt to their disease because it encourages them to accept treatment with a positive attitude. Therefore, this study infers that cirrhotic patients who are single tend to adopt emotion-oriented coping behavior because their support systems are less favorable than those of the married patients.

Compared with patients who are employed, unemployed patients with cirrhosis adopt emotion-oriented coping behavior more frequently. This phenomenon was similar to that observed by Liou et al. [9]. This result may be attributable to the exclusion of older people (aged 65 and over) in the present study and inclusion of middle-aged participants ( $n=67 ; 88.2 \%$; aged $40-63$ years). Lewisk [21] observed that losing a job is a stressful event in life because it deprives people of the ability to control the most crucial aspect of life. In addition, Tseng [22] indicated that middle-aged men are mostly the breadwinner of the family and therefore being unemployed implies loss of stable income, which causes material impact on their family. In the long run, patients would feel insecure about their financial and living conditions. Moreover, patients in their middle age most often experience stress from work and family; thus, without a support system, these patients tend to perceive negative emotions and become incapable of coping with stress [23]. Accordingly, this study infers that unemployed patients with cirrhosis tend to adopt emotion-oriented coping behavior compared with those who are employed.

The present study showed that participants experiencing fatigue engaged in emotion-oriented coping behavior more often than those without fatigue did. According to Tsao [19], higher disease severity (i.e., higher Child-Pugh level) induces fatigue in patients with cirrhosis. Patients with high disease severity experience more complications and a poor sense of control over their symptoms. They also perceive a higher level of physical discomfort. Furthermore, these patients tend to feel anxious and worry that their disease condition will deteriorate further. Therefore, this study infers that cirrhotic patients who experience fatigue have a higher level of disease severity, which induces them to adopt emotion-oriented coping strategy.

Frequency of alcohol intake was significantly and positively correlated with emotion-oriented coping behavior. During the interviews, the authors of this study learned that patients with cirrhosis drank alcohol-containing energy beverages to stay awake and energize themselves at work. Occasionally, they drank alcohol to aid sleep or alleviate stress and forget about their worries in work and life. Hasin, Stinson, Ogburn, and Grant [24] also reported that people who abuse alcohol demonstrate unstable work performance. In the present study, the participants were mostly aware that abstinence from alcohol can delay disease progression. However, if they stopped drinking, the participants tend to experience problems such as shakiness and poor sleep quality, which prompted them to take sedatives or sleeping pills. As a result, the need to take medication lowered their intention and persistence to quit drinking. In this study, 60 out of the 76 participants (78.9\%) drank alcohol habitually, and only 12 of them (21.1\%) quit drinking. Most of the patients mentioned that they usually drink to forget their troubles temporarily. Accordingly, this study infers that cirrhotic patients who drink alcohol tend to adopt emotion-oriented coping behavior.

In this study, disease severity was significantly and positively correlated with the frequency of adopting emotion-oriented coping behavior. This result was possibly attributed to two reasons. The first reason is that most of the participants who used emotion-oriented coping strategy exhibited a moderate or high level of disease severity $(n=41 ; 54 \%)$. This indicated that most patients experienced multiple complications and health problems. In addition, Liao, Yang, $\mathrm{Ku}$, Hsiao, and Chiang [25] observed a significantly positive correlation between symptom distress and uncertainty. The other reason is based on a study by Mishel [3], which asserted that patients who use emotion-oriented coping strategy tend to escape from their problems in reality and abuse alcohol. It was hypothesized that patients who are emotionally unstable tend to mitigate their stress by drinking alcohol, which has been regarded as a means of escaping from problems. Therefore, this study infers that patients whose disease is in a severe state are often emotionally unstable because of their symptom distress, which stimulates them to engage in emotion-oriented coping behavior more frequently.

\section{Correlation between personal characteristics and problem- oriented coping behaviors}

This study determined that problem-oriented coping behavior was adopted more frequently by unemployed patients with cirrhosis than by those who are employed. Few studies have discussed the coping behaviors of patients with cirrhosis. Therefore, the present study selected patients with different diagnoses as the target of comparison and discussion. Liou et al. [9] investigated the coping behaviors of patients receiving dialysis and observed that patients who have a job often engage in problem-oriented coping behavior, whereas their counterparts tended to exhibit emotion-oriented coping behavior. The reason why the results of the present study differed from those of Liou et al. [9] can be explained as follows. Unemployed patients are not concerned about discontinuing working because they have to be hospitalized for treatment. However, not working temporarily may influence their way of living because they would have no stable source of income. According to Leana and Feldman [26], the coping behaviors of unemployed people usually include seeking family and friends' 
support, finding another job, or lowering the standards of target jobs. Miller [4] noted that problem-oriented coping behavior can help individuals proactively confront problems, find solutions, and gain a sense of control in face of threats. The present study determined that frequency of alcohol intake by patients with cirrhosis correlated significantly and positively with problem-oriented coping behavior. This is possibly because physicians typically inform patients that abstinence from alcohol could effectively relieve symptoms and delay disease progression. Therefore, these patients would adopt problemoriented coping strategy by attempting to quit drinking or drink as seldom as possible. Accordingly, this study infers that unemployed patients with cirrhosis more often engage in problem-oriented coping behavior than do patients who have a job.

The results of this study showed that adopting problem-oriented coping behavior was more frequent among cirrhotic patients with a high level of education than among those with a low level of education. This result accords with that of Liou et al. [9] and Wu, Lai, Sheu, and Shun [27], both of which have indicated that patients who are educated typically adopt problem-oriented coping strategies. A possible explanation could be that educated people are inclined to ask and clarify questions if they are unclear about the causes of symptoms. Furthermore, people with a high level of education can access and understand disease-related information more easily than do those with a low level of education [28]. Therefore, this study infers that the education level of cirrhotic patients correlates positively with the frequency of adopting problem-oriented coping behavior.

\section{Correlation between uncertainty and coping behaviors}

The present study indicated that when the level of uncertainty among cirrhotic patients was high, the frequency of adopting emotion-oriented and problem-oriented coping behaviors increased. Few studies have explored the relationship between uncertainty and coping behaviors in patients with cirrhosis. Therefore, the authors of this study compared the results of previous studies that have focused on patients with chronic disease. The comparison showed that past studies have generated inconsistent results. For example, Sheu [10] indicated that inpatients with myocardial infarction who perceived high level of uncertainty have the tendency to adopt emotionoriented coping behavior. By contrast, those who perceived low level of uncertainty have the tendency to adopt problem-oriented coping behavior. Examining 128 patients with liver diseases (e.g., hepatitis, liver cirrhosis, and liver cancer), Ho et al. [29] found that the level of uncertainty in these patients correlated negatively with problemoriented coping behavior and positively with emotion-oriented coping behavior. These results differed from those obtained by the present study. In addition, the two aforementioned results were similar to those of Sheu et al. [30], who reported that uncertainty in patients with myocardial infarction correlated significantly and positively with their emotion-oriented coping behavior but non-significantly with problem-oriented coping behavior. This finding can be further supported by the argument of Krumm [31] that individuals adopt either problem-oriented or emotion-oriented coping behavior when facing stressful situations. This argument can support the perspective of Lazarus and Folkman [32] that people can independently adopt either emotion-oriented or problem-oriented or both types of coping behaviors simultaneously. During the interviews, the authors of the present study determined that the patients coped with stressful situations (e.g., when experiencing symptom distress) either by adopting problem-oriented approach (search for disease-related information or use different methods to solve problems) or by using emotion-oriented methods (such as being emotionally unstable, crying, escaping from reality, blaming others, or refusing to change).

\section{Conclusion}

According to the results of this study, patients with cirrhosis perceived a moderate or high level of uncertainty. A high level of uncertainty was associated with a lack of religious beliefs, high disease severity, and feelings of fatigue. The participants of this study predominantly used problem-oriented coping strategy to cope with the disease. Emotion-oriented coping behavior was more frequently observed in participants who were single, unemployed, or experienced fatigue. Frequency of alcohol intake and disease severity correlated positively with emotion-oriented coping behavior. Unemployed participants tended to use problem-oriented coping behavior. Education level and frequency of alcohol intake were positively correlated with problemoriented coping behavior. Level of uncertainty correlated positively with emotion-oriented and problem-oriented coping behaviors.

\section{Limitations and Suggestions}

This study had three limitations regarding the research samples and process: (a) Sampling was confined to the hepatobiliary and gastroenterology ward of a regional hospital in Southern Taiwan. Therefore, the results could not be generalized to all patients with cirrhosis in Taiwan. (b) This study involved a cross-sectional correlational research design; thus, the researchers evaluated only the patients' conditions at the time of recruitment. (c) Because of limited human resources and time constraints, this study could not use random sampling to recruit participants. Although the recruitment period lasted 8 months, only 76 samples were obtained, which could indicate a lack of representativeness.

This study proposed the following suggestions for future reference. First, the concepts of uncertainty and coping behaviors could be included in medical-surgical nursing courses regarding diseaserelated psychosocial concepts and nursing. Thus, nursing students can acquire a basic understanding of these concepts. During their medical-surgical internships, nursing students can learn how to use uncertainty and coping behaviors scales in evaluating patients withcirrhosis. Second, case management models for the care and follow-up of patients with cirrhosis can be developed to evaluate the effects of the disease and track the improvement of disease complications. Third, future researchers can adopt a longitudinal research design to determine the influences of uncertainty on the coping behaviors of patients with cirrhosis. Moreover, random sampling can be performed to enhance sample generalizability.

\section{Implications for practice}

By using uncertainty and coping behavior scales, clinical nurses can improve their ability to detect the uncertainty patients with cirrhosis have toward their disease. Furthermore, the scale can be used to ascertain why patients experience feelings of uncertainty and what coping strategies they adopted. These results can assist nurses in identifying the level of uncertainty in cirrhotic patients and detecting their psychological problems to mitigate the patients' negative emotions through early interventions. Since cirrhotic patients frequently exhibit emotion-oriented coping behaviors because of their high uncertainty, nurses should encourage them to express their emotions and problems, and listen to them patiently. If patients were high levels of uncertainty, frequently exhibit problem-oriented coping 
Citation: Lin WJ, Huang HM, Lee BO, Sun FK (2017) The Relationships Between Uncertainty and Coping Behaviors of Patients With Liver Cirrhosis. Int J Nurs Clin Pract 4: 261. doi: https://doi.org/10.15344/2394-4978/2017/261

Page 7 of 7

behaviors, by spontaneously providing disease-related information and social support, they can readily adopt to their disease conditions.

\section{Competing Interests}

The authors declare that they have no competing interests.

\section{Author Contributions}

WJL, HMH are responsible for the idea behind this study and its structure, as well as collected all the data and the data analysis. $\mathrm{HMH}, \mathrm{BOL}$, and FKS are responsible for the literature review and discussion part of this paper and its structure. All authors developed the preparation of the manuscript. All authors have agreed on the final version.

\section{Research Ethics}

Ethics approval to conduct this study was obtained from the study hospital's institutional review board (IRB number: 14-015) in Taiwan.

\section{Informed Consent}

All 76 participants were signed the Consent Form as evidence of informed consent. To protect participants' privacy, all identifying information was removed and replaced with a code number in the process of analysis.

\section{References}

1. Ministry of Health and Welfare (2013) 2012 Annual Statistical Yearbook of the cause of death.

2. Chen YC, Tsai SL, Tsai CH, Huang S (2012) Adjusting experience of patients with liver cirrhosis ascites. New Taipei Journal of Nursing 14: 1321.

3. Mishel MH (1981) The measurement of uncertainty in illness. Nurs Res 30: 258-263.

4. Miller JF (1992) Coping with chronic illness, Philadelphia: Davis, pp.413433.

5. Ko NY, Shu ST (2005) Informational needs, health locus of control and uncertainty among women hospitalized with gynecological diseases. Chang Gung Medical Journal 28: 559-566.

6. Lai HL, Lin SY, Yeh SH (2007) Exploring uncertainty, quality of life and related factors in patients with liver cancer. Hu Li Za Zhi 54: 41-52.

7. Sheu SL, Hwang SL (1996) Validation of Chinese version of Mishel's Uncertainty in Illness Scale. The Journal of Nursing Research 4: 59-68.

8. Yang HL, Chang SN (2007) An experience nursing an uncertain patient with recurrent cervical cancer. The Journal of Nursing 54: 101-105.

9. Liou LJ, Huang ML, Hung LC (2008) Coping behaviors and outcomes in hemodialysis patients. Nephrology Nurses association of Taiwan 7: 34-49.

10. Sheu SL (2001) Uncertainty and anxiety in patients with initial attack of myocardial infarction: The effect of coping methods. The Journal of Nursing Research 9: 159-171.

11. Hwu YJ, Lin FY (2005) An empirical study of chronic impact, health behaviors, and coping outcomes in people with chronic illness. Journal of Evidence-Based Nursing 1: 140-148.

12. Tsui WY, Lin KC, Huang HC (2013) An investigation of body image changes and coping behaviors in oral cancer patients following surgery. Journal of Nursing Healthcare Research 9: 127-138.

13. Chiou CP (1987) A hemodialysis patients of stress, coping behavior and social support. The Journal of Nursing 34: 55-61.

14. Lu YC, Yen WJ, Lee S (2013) Exploring the uncertainty and coping strategies of parents of preterm infants. Journal of Nursing and Healthcare Research 9: 23-33.

15. Martin LM, Dan AA, Younossi ZM (2006) Measurement of health-related quality of life in patients with chronic liver disease. Liver Transplantation 12: $22-23$
16. Yang BJ, Chiu PR, Shih FY (2008) Uncertainty about disease for postoperation oral cancer patients upon discharge. Fu-Jen Journal of Medicine 6: 53-68.

17. Ruth A (2006) Spirituality and family nursing: Spiritual assessment and interventions for families. Journal of Advanced Nursing 53: 287-294.

18. Lin $\mathrm{YH}$, Liou SH, Chen $\mathrm{CH}$ (2008) Spiritual care in nursing practice. The Journal of Nursing 55: 69-74.

19. Tsao MC (2004) Fatigue and sleep quality in cirrhotic patients. Unpublishedmaster's thesis. Taipei city, Taipei Medical University: Department of Nursing.

20. Zheng YM, Hsiao YS, Chang HJ (2012) The nursing experience of an elderly man living alone with ineffective individual coping induced by liver cirrhosis combined with esophageal varices hemorrhage. Medicine Journal of Show-Chwan 11: 129-136.

21. Lewisk K (1983) Grief in chronic illness and disability. Journal of Rehabilitation 49: 8-11.

22. Tseng $\mathrm{YH}(2004)$ Health behaviors of middle-aged adults. The Journal of Nursing 51: 20-24.

23. Pan PH, Rong JR, Wu SI (2013) The study of health problems and selfefficacy in health behaviors influence the mental health of middle-aged people. The Journal of Psychiatric Mental Health Nursing 8: 12-22.

24. Hasin DS, Stinson FS, Ogburn E, Grant BF (2007) Prevalence, correlates, disability, and comorbidity of DSM-IV alcohol abuse and dependence in the United States. Archives of General Psychiatry 64: 830-842.

25. Liao HC, Yang CY, KuYL, Hsiao YL, Chiang MC (2005) Uncertainty and its related factors for liver cancer patients. Chang Gung Nursing 16: 252-263.

26. Leana C, Feldman DC (1988) Individual responses to job loss: Perceptions, reactions, and coping behaviors. Journal of Management 14: 375-389.

27. Wu RJ, Lai YH, Sheu JC, Shun SC (2015) The relationship between coping strategies and type $D$ personality in non late stage hepatocellular carcinoma survivors. Journal of Cancer Research Practice 2: 213-223.

28. Yang HC (2010) The relationship among health locus of control,ay of coping, and Bio-psycho-social adaptation effect on the head and neck cancer patients: A longitudinal Study. Unpublished doctor's thesis. Taipei city, National Taiwan Normal University: Department of Educational Psychology and Counseling (published in Chinese)

29. Ho HY, Chan AY, Wu HF, Chung YC, Chen LH, Huang HF (2006) Exploring uncertainty and associated factor of patients with liver disease. Paper presented at the Symposium of Health and Management, Taiwan, Taipei city.

30. Sheu SL, Hwang SL, Lin HS (1998) Perception of uncertainty and coping behaviors of hospitalized patients with acute myocardial infarction. Taiwan Medical Journal 2: 498-508.

31. Krumm S (1992) Psychosocial adaptation of the adult with cancer. Western Journal of Nursing Research 14: 729-737.

32. Lazarus RS, Folkman S (1984) Stress, appraisal, and coping. New York, NY: Springer. 